\title{
VARIACIÓN EN LAS PROPIEDADES BROMATOLÓGICAS DE TRES TIPOS DE PASTURAS PARA USO GANADERO EN EL DISTRITO DE CONSERVACIÓN SUELOS BARBAS BREMEN
}

\author{
VARIATION IN THE BROMATOLOGICAL PROPERTIES OF THREE TYPES \\ OF PASTURES FOR LIVESTOCK USE IN THE BARBAS BREMEN SOILS \\ CONSERVATION DISTRICT
}
Henry Reyes Pineda ${ }^{1 *}$, Rafael Guacán-Andrade ${ }^{2}$, Felipe Narváez ${ }^{3}$, Juan Car- los Rengifo ${ }^{4}$, Leidy Carolina Cardona Hernández ${ }^{5}$, Marcelino Galvis ${ }^{6}$ y Sandra Milena Manrique ${ }^{7}$

1. Facultad de Ciencias Agroindustriales, Universidad del Quindío.

Mail: hreyes@uniquindio.edu.co

2. Facultad de Biología, Universidad del Quindío. Mail: camilo8920@gmail.com

3. Facultad de Biología, Universidad del Quindío. Mail: skarny_92@hotmail.com

4. Facultad de Ingeniería Mail: juan.c.rengifo@redcampo.com

5. Grupo de investigación en Ciencias ambientales. Universidad del Quindío

Mail: lccardona@uniquindio.edu.co

6. Facultad de Ciencias Agroindustriales. Universidad del Quindío

Mail:megalvis@uniquindio.edu.co

7. Corporación Regional del Quindío, CRQMail: sandramanrique@gmail.com

*Correspondencia del Autor: Henry Reyes Pineda email: hreyes@uniquindio.edu.co

\section{RESUMEN}

El Municipio de Filandia está ubicado en la vertiente occidental de la Cordillera Central de Colombia, cuenca del Alto Cauca. Su territorio representa el 5.58\% de la extensión total del Departamento del Quindío; dicho municipio presenta una gran actividad ganadera la cual ha venido en aumento, siendo la ganadería de doble propósito (leche y carne) como actividad económica más utilizada para el sustento de sus hogares. Se realizó una recolección de muestras de pastos y tierras de propiedades de miembros de asociaciones ganaderas, como parte de la estrategia integradora del departamento que promueve las técnicas menos dañinas para el medio ambiente por la actividad ganadera. Encontramos que los valores nutricionales de las pasturas tienen niveles de proteína media y valores de grasa bajos con relación a los marcos de referencia mínimos. Esto puede ser atribuido a los periodos de altas lluvias que se observan en la región y a los procesos de ganadería no estandarizados y controlados, pues en dichos predios no se realiza rotación, siendo esto último un mecanismo que permite el máximo aprovechamiento del potrero por unidad de ganado.

Palabras claves: Suelos, análisis bromatológico, área protegida, ganadería

Cómo citar:

Reyes et al. (2017) VARIACIÓN EN LAS PROPIEDADES BROMATOLÓGICAS DE TRES TIPOS DE PASTURAS PARA USO GANADERO EN EL DISTRITO DE CONSERVACIÓN SUELOS BARBAS BREMEN. Revista de Investigaciones de la Universidad del Quindío. Vol 29, pp.53-58.

Información del artículo: Recibido: 02 febrero 2018; Aceptado: 23 agosto 2018

Revista de Investigaciones de la Universidad del Quindío, 29(1). pp 53-58; 2017.

ISSN: 1794-631X (impreso) ISSN: 2500-5782 (en línea) 


\begin{abstract}
.
The Municipality of Filandia is located on the western slope of the Central Cordillera of Colombia, Alto Cauca basin. Its territory represents $5.58 \%$ of the total extension of the Department of Quindío. This municipality has an increased livestock activity with dual purpose (milk and meat) as the most important economic activity for the sustenance of families. We studied the agricultural practices of the members of the livestock association of the municipality and we also performed a bromatological analysis of pastures and lands, in order to know their quality as part of the department's integrative strategy that promotes the least harmful techniques of livestock activity for the environment. We found that the nutritional values of the pastures have medium protein levels and low fat values in relation to the minimum reference frames. This can be attributed to the high rainfall periods witnessed in the region and to the non-standardized and controlled livestock processes, since in such farms there is no rotation, this being the cause of maximum use of the paddock per unit of livestock.
\end{abstract}

Keywords. Soils, bromatological analysis, cattle.

\section{INTRODUCCIÓN.}

El municipio de Filandia se caracteriza por tener una buena parte del territorio en el piedemonte de la cordillera Central y en el sur occidente de la parte Norte del Valle del Cauca, más concretamente al límite de los municipios de Alcalá y Ulloa. La zona rural del municipio muestra un área expuesta a la agricultura comercial, donde se registra un dinamismo alrededor de cultivos de café, banano, forestales, cultivos de tomate y flores bajo invernadero, registrándose así un acelerado proceso de cambios en el uso del suelo. Las actividades pecuarias son de igual forma representativas en la economía del Municipio y las que más sobresalen son la ganadería intensiva y extensiva, la porcicultura, la piscicultura y la avicultura, hecho que viene causando una serie de impactos sociales, económicos y ambientales, toda vez que el cambio en el uso del suelo de café a ganadería deforesta un vasto sector de las praderas.

La importancia de las pasturas en la ganadería radica en que constituye la dieta básica y más económica en la alimentación de rumiantes (bovinos, caprinos y ovinos), además proporcionan materia orgánica al suelo, lo que ayuda en la conservación protegiendo de la erosión (Cabrera, 2002). Por otra parte, las especies establecidas en la pradera deben estar en buen estado, creciendo con vigor y libre de plagas y enfermedades antes de ser utilizadas, de lo contrario se contribuirá a acelerar el deterioro y degradación de estas (Franco et al. 2006).

El género Brachiaria tiene características adecuadas de rendimiento, que junto a la capacidad de adaptación a suelos ácidos contribuye a incrementar los rendimientos productivos de la ganadería (Olivera et al., 2008). Alvarado et al., (1990) encontraron que Brachiaria decumbens, posee un gran potencial en producción de materia seca y excelentes características adaptativas para suelos pobres de sabanas, determinando contenidos de proteína relativamente altos de 9.4 y $8.8 \%$ para 42 y 56 días de edad. Sin embargo, se indica que la calidad nutritiva de un pasto cambia de acuerdo con las condiciones ambientales y del manejo integral que reciban (Miles, 2006). La Brachiaria decumbens se adapta a una amplia gama de suelos de fertilidad variable y textura desde arenosos a arcillosos; pero exige terrenos bien drenados (Metidiare, 1988).

Trujillo (1986), menciona que en la introducción a Colombia la Brachiaria decumbens, ha demostrado una buena adaptación a suelos de baja fertilidad natural, y actualmente es una de las gramíneas más utilizadas en explotaciones ganaderas localizadas a alturas inferiores a 1000 m.s.n.m.

Cynodon nlemfuensis conocido como pasto estrella africana es un pasto tropical perenne de clima caliente, cuyo crecimiento por medio de estolones a menudo leñosos, le permite distribuirse rápidamente al generar raíces profundas y culmos de hasta $1 \mathrm{~m}$ de altura que al mismo tiempo producen semillas que facilitan su dispersión (Mislevy 2002, Cook et al. 2005). Es más suave, palatable y digestible que el pasto bermuda (Cynodon dactylon) y es susceptible al clima frío (Burton 1993). Es por ello que el objetivo de esta in- 
vestigación consiste en analizar las propiedades bromatológicas de tres tipos de pasturas provenientes del área protegida del Distrito de Conservación Barbas Bremen, como parte integral del uso del suelo, dentro del proyecto de regalías desarrollado en forma interdisciplinaria con la Corporación Regional del Quindío, CRQ y RedCampo.

\section{MATERIALES Y MÉTODOS}

Para la disposición de muestras de pastos se llevó a cabo una toma sistemática de muestras de pasturas, en los predios ganaderos asociados al Distrito de Conservación de Suelos Barbas Bremen del municipio de Filandia y Circasia, municipios que se encuentran ubicados al norte del Departamento del Quindío a los $04^{\circ} 45^{\prime}-04^{\circ} 37^{\prime} \mathrm{N}$ y $75^{\circ} 41^{\prime}-75^{\circ} 32^{\prime} \mathrm{W}$, entre 1650 y 2500 metros sobre el nivel del mar (msnm), en los ramales occidentales de la cordillera central (Franco \& Bravo 2005). Los análisis bromatológicos se llevaron a cabo en los laboratorios de investigación de la Facultad de Ciencias Agroindustriales, tomando como referencia el protocolo y recomendaciones del laboratorio de post-cosecha de la Universidad del Quindío, el cual indica realizar cortes a la altura de 10 $\mathrm{cm}$ sobre la raíz mediante el uso de tijeras podadoras. Las muestras fueron homogeneizadas y pesadas en campo, siendo 200 gramos la muestra requerida para dicho análisis. Seguido a esto las sub-muestras fueron empacadas en bolsas de papel, las muestras empacadas se llevaron al horno del CIBUQ a una temperatura de $70^{\circ} \mathrm{C}$, para su respectivo secado (Van Soest, 1994), por un periodo de 24 horas, esto con el fin de obtener la materia seca. Posteriormente se analizó la cantidad de grasa y cenizas, se determinó el peso total de las pasturas y su porcentaje de pérdida de agua.

Técnicas de análisis experimental: Debido a la amplia información recolectada, se utilizó un análisis experimental de inferencia estadística, ya que se tiene como objetivo la estimación de las propiedades o características de una zona a partir del análisis de una muestra de dicha población. Los datos fueron analizados, mediante análisis de varianza, utilizando el software Statgraphics.

\section{RESULTADOS}

La Figura 1 muestra los porcentajes de proteína, los cuales se encuentran entre los valores medios del valor de referencia, a la vez, la proteína en las pasturas otorga biomasa en los animales repercutiendo también en su producción de leche.

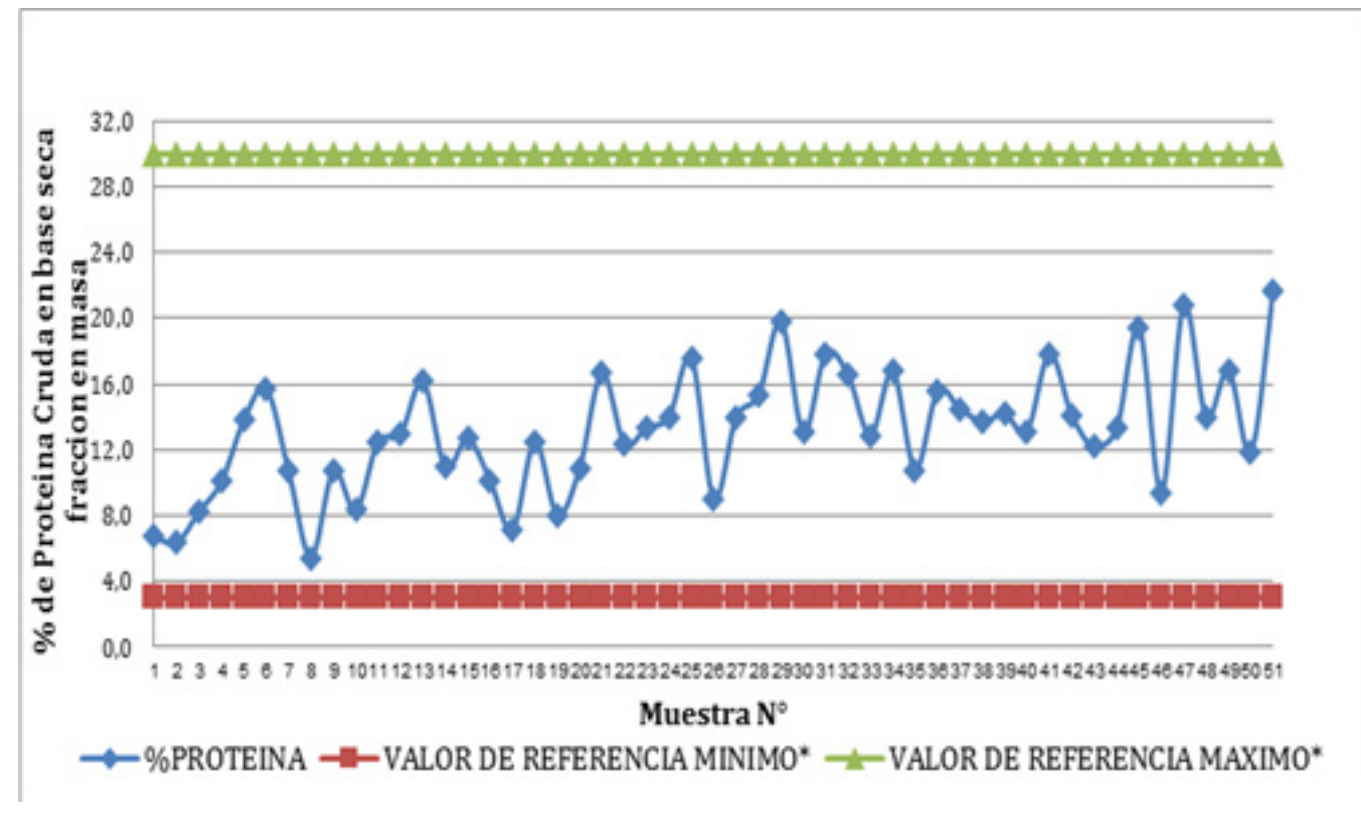

Figura 1. Proteína presente en las muestras de pastos de los predios seleccionados.

En cuanto a los valores de grasas, tal como se muestra en la Figura 2, se encontró que son bajos, ya que no alcanzan los valores mínimos de referencia; estos valores pueden verse reflejados por la época de altas lluvias las cuales por escorrentía limitaron el aprovechamiento de los periodos de fertilización. 


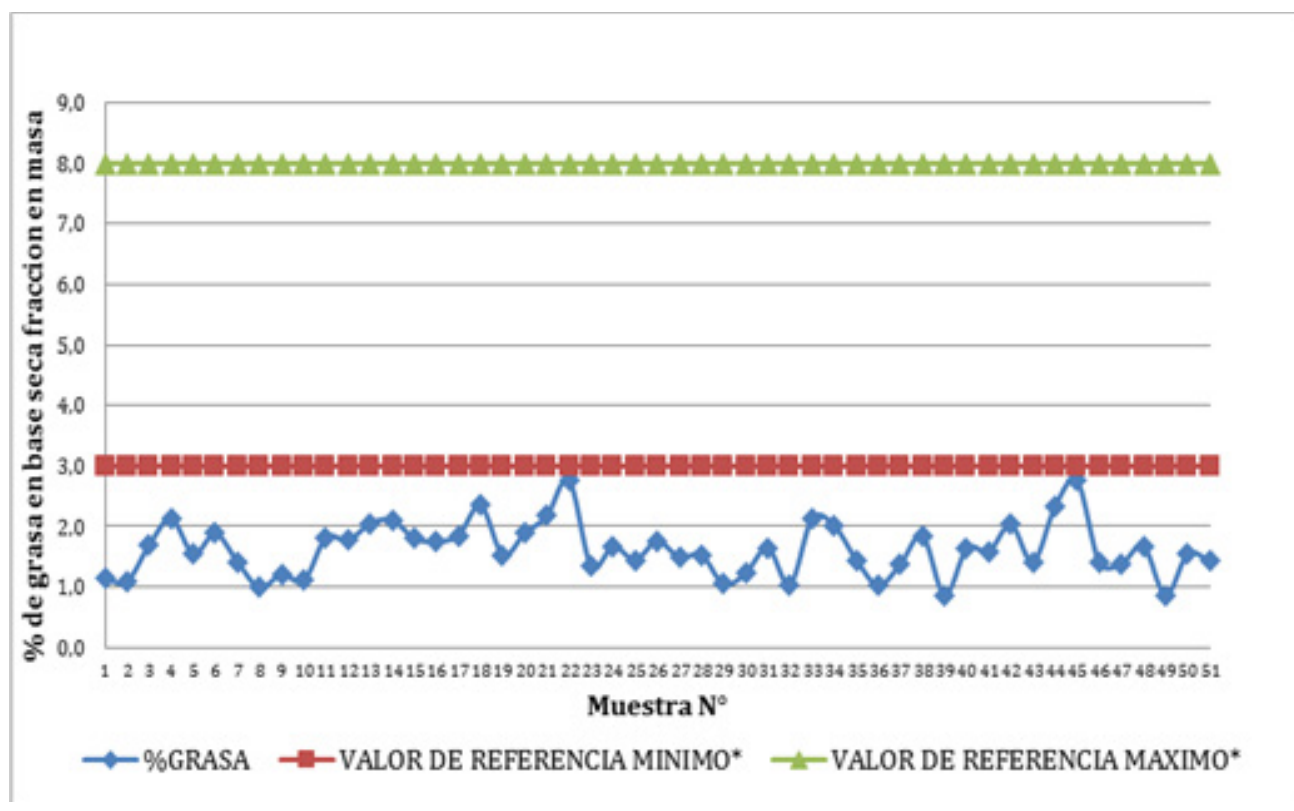

Figura 2. Grasa presente en las muestras de pastos de los predios seleccionados.

En cuanto a los materiales resultantes como la ceniza los valores se comportaron con tendencia a los niveles bajos, que tal como se expresó anteriormente están directamente relacionados con procesos físicos de obtención de nutrientes por ausencia de estos, siendo el agua el principal implicado ya que por escorrentía filtraba los abonos tanto orgánicos como inorgánicos aplicados.

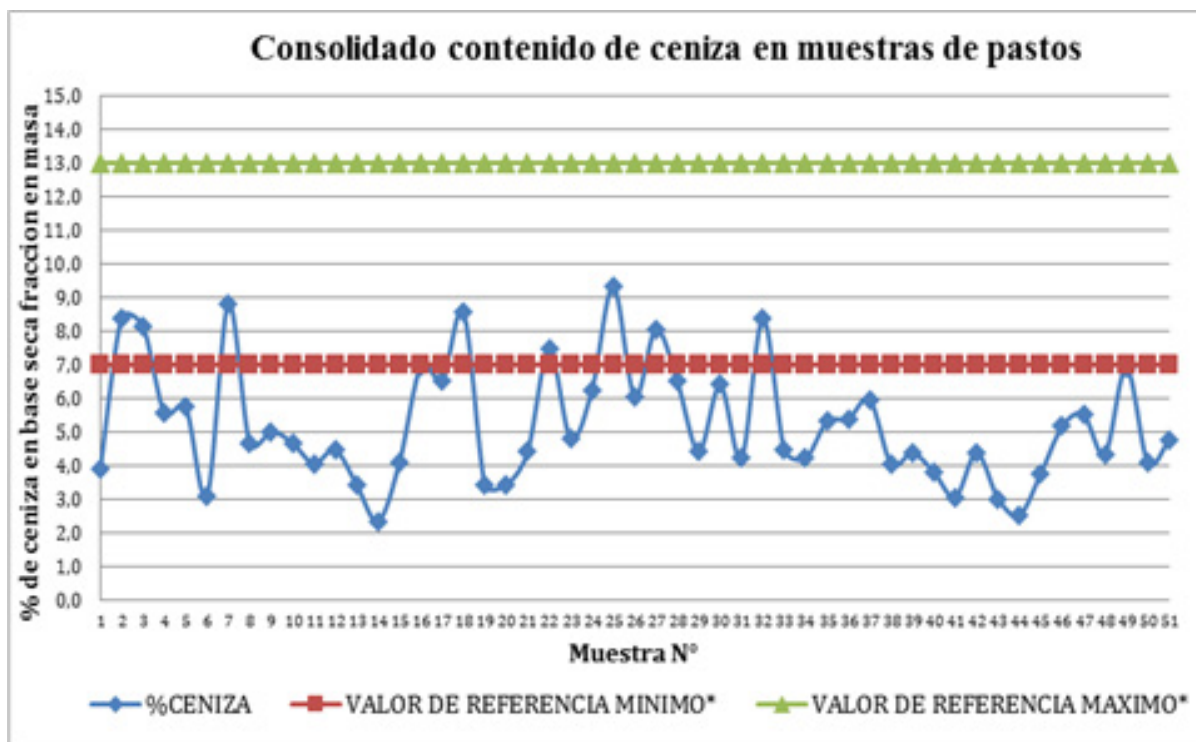

Figura 3. Ceniza presente en las muestras de pastos de los predios seleccionados.

El comportamiento presentado en el contenido de fibra cruda, mostrado en la Figura 4, es normal, ocasionado al uso del suelo y oscilando entre valores de 2 a
8 , los cuales son característicos en la reserva Barbas Bremen. 


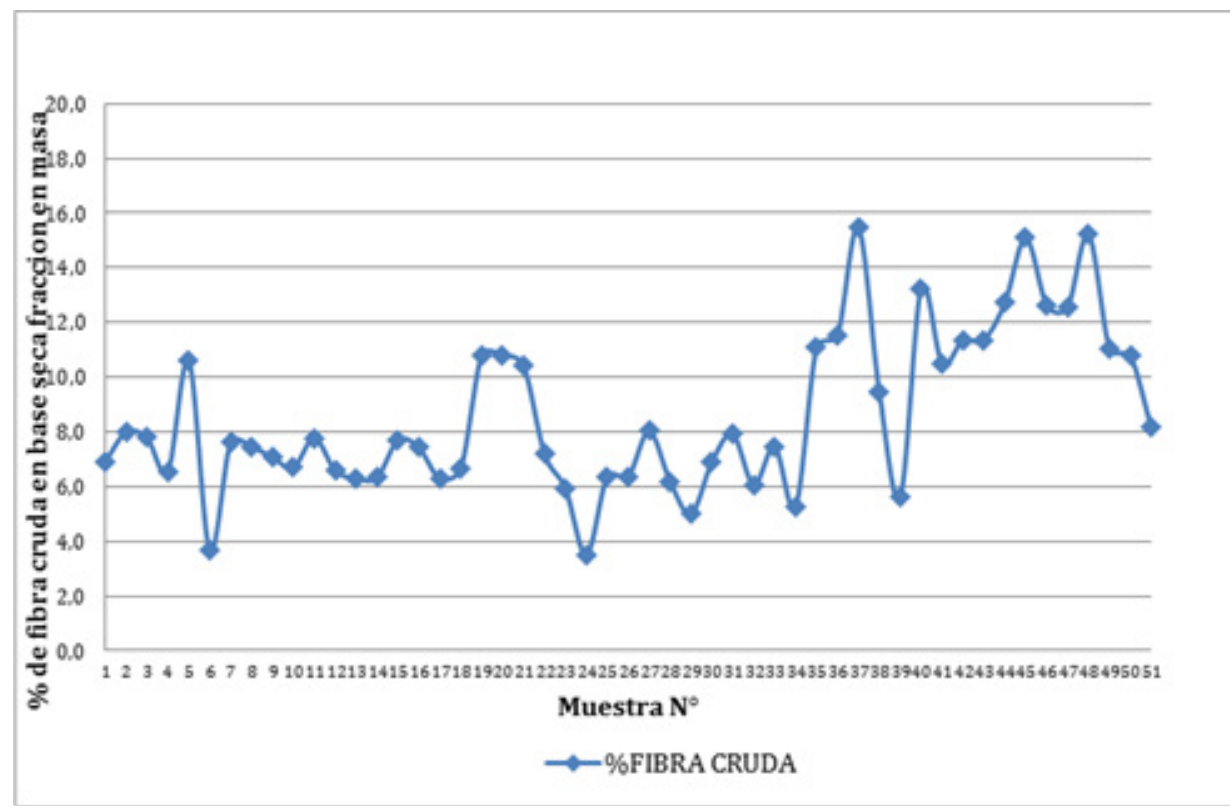

Figura 4. Fibra presente en las muestras de pastos de los predios seleccionados.

El contenido de humedad en las tres muestras de pastos es representativo para el tipo de suelo en esta área protegida que cuenta con una buena reserva hídrica, que pese al uso indiscriminado del suelo, son valores cercanos a los esperados, tal como puede reflejarse en la Figura 5.

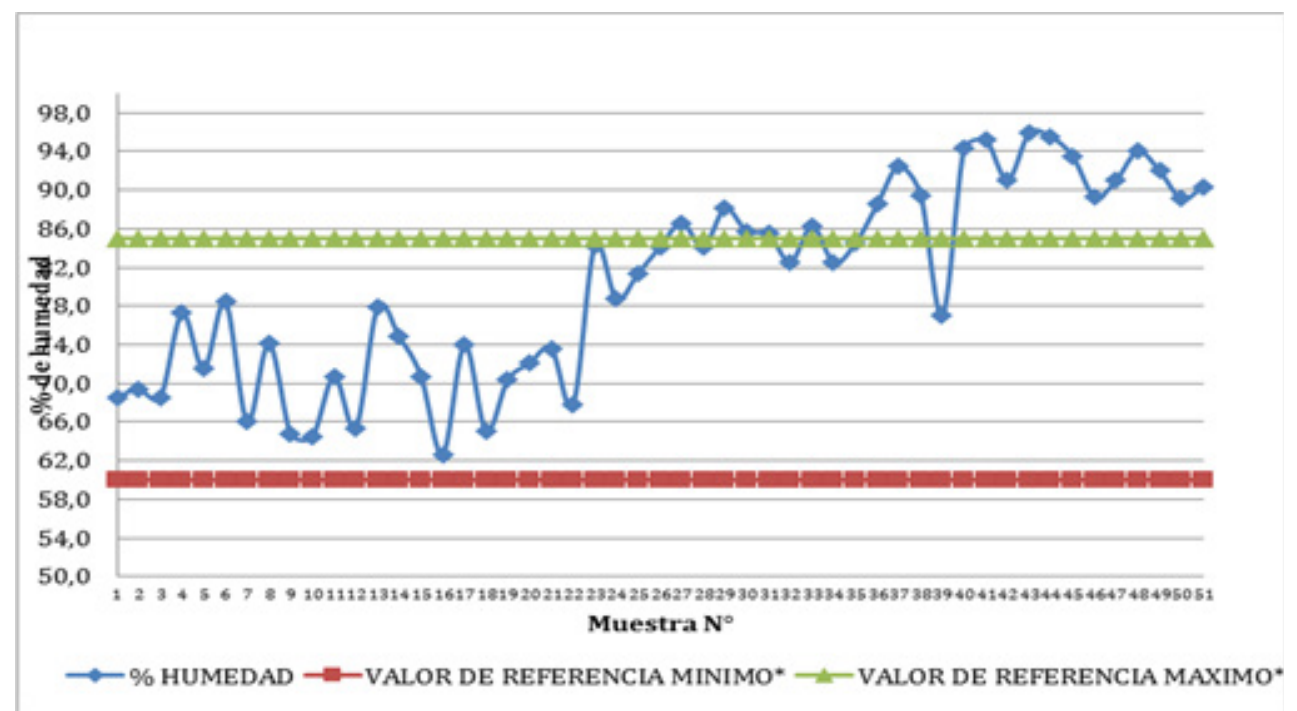

Figura 5. Humedad presente en las muestras de pastos de los predios seleccionados.

\section{DISCUSIÓN}

Los valores nutricionales obtenidos por los análisis bromatológicos de las pasturas muestran unos niveles de proteína que escalan los valores intermedios-bajos con referencia al nivel de máximo y mínimos planteado por Molano (2012). Estos valores se comportan de dicha manera debido a las condiciones ambientales presentes en la zona la cual al estar en una temporada de altas lluvias expresan gran cantidad de humedad relativa representada en el peso seco de las muestras, perdiendo entre el 70 y $80 \%$ de su peso en fresco. También estos resultados expresan un comportamiento en el consumo del pasto, ya que la gran mayoría de campesinos no aplicaban los sistemas de rotación haciendo que no se optimizara el consumo de pasto en su mejor momento, permitiendo lignificarse y así disminuyendo su valor nutricional. En relación con los resultados de ceniza, se debe tener en cuenta que la ceniza es el resultado del proceso de eliminación de agua, grasas y proteína presentes en un alimento, por lo cual los resultados encontrados en la presente 
investigación, al ser comparados con los valores de humedad, nos revelan que grandes porcentajes de las muestras estaban constituidos por agua.

El desarrollo tecnológico de los suelos es una estrategia de acondicionamiento que permite la optimización de los mismos para brindar unos niveles de nutrientes más elevados a la hora de cosechar la pradera esto va de la mano con el tiempo de siembra y el estado del año, pues dichos factores se relacionan con el aprovechamiento de la planta para la translocación de nutrientes (Méndez, 2016).

Existen factores importantes que de una $\mathrm{u}$ otra forma alteran los resultados obtenidos, tal como lo es el clima, la topografía de algunos sitios de muestreo, y el tipo de uso del suelo, que en algunas veredas está dedicado exclusivamente a la ganadería y en otros a usos silvo pastoriles. De aquí que, en futuros estudios, debe tenerse en cuenta otros usos del suelo, como lo es el turístico, ya que el ambiente humano ocasiona delicados problemas medio ambientales en esta área protegida. Por otra parte, la cantidad de predios analizados es un buen referente y servirá de guía para futuros estudios para las corporaciones y entidades gubernamentales, que serán en últimas quienes deben tomar acciones correctivas con el uso indiscriminado del suelo.

AGRADECIMIENTOS. Este trabajo hace parte de un proyecto macro financiado por el Departamento Nacional de Planeación, DNP, Colciencias, la Gobernación del Quindío, la Corporación Regional del Quindío, la Universidad del Quindío y RedCampo, por lo cual, los autores agradecen por toda la colaboración y aportes financieros.

\section{BIBLIOGRAFÍA}

1. Alvarado, A.; Arriojas, L.; Chacón, E.; Rodríguez, S. y Chacín, F. 1990. Estudios sobre henificación del pasto Barrera (Brachiaria decumbens Stapf) en condiciones de sabanas del Piedemonte Barinés I. Producción y valor nutritivo de la materia seca. Zootecnia Tropical. 8(1-2):17-36.

2. Cabrera, D. 2002. Manejo y uso de pastos y forraejes en ganadería tropical.

3. Cook B.G., Pengelly B.C., Brown S.D., Donnelly J.L., Eagles D.A., Franco M.A., Hanson J., Mullen B.F., Partridge I.J., Peters M., Schultze R. 2005. Tropical Forages: an interactive selection tool. [CDROM]. CSIRO, DPI\&F (Qld), CIAT and ILRI, Brisbane, Australia.

4. Franco, A. \& Bravo, G. (2005) Áreas importantes para la conservación de las aves en Colombia. Pp 117-281 in BirdLife International \& Conservation International. Áreas Importantes para la Conservación de las Aves en los Andes Tropicales: sitios prioritarios para la conservación de la biodiversidad. Quito, Ecuador: BirdLife International (BirdLife Conservation Series No. 14).

5. Franco, Q.; Calero, Q.; Duran, C. (2006). Manejo y utilización de forrajes tropicales multipropósito. Impresión: Paola A. Sanmiguel P. Palmira - Valle del Cauca - Colombia. P. 32.

6. Méndez, L. Lilia. (2016). Retención de alimentos. Tecnología Investigación y Desarrollo, pp. 25-29

7. Miles, J. W. 2006. Mejoramiento genético en Brachiaria. Objetivos estratégicos, logros y proyección. Pasturas Tropicales. (28):1-26.

8. Mislevy p. 2002. Stargrass. Florida Cooperative Extension Service, Institute of Food and Agricultural Sciences, University of Florida, Gainesville, USA. 4 p.

9. Olivera, Y.; Machado, R.; del Pozo, P.; Ramírez, J. y Olivares J. 2008. Persistencia del pastizal en una colección de Brachiaria spp., en un suelo ácido. Pastos y Forrajes. 31(4):333.

10. Van Soest, P. (1994). Nutritional Ecology of the Ruminant. Cornell University Press, 476 pp. Ithaca (USA). 